\title{
Challenges and Strategies for Conducting Clinical Research during the COVID-19 Pandemic: Experiences from Resource Limited Settings
}

\author{
Achan Jane, Asadu Serwanga, Aanyu T. Hellen, Opigo Jimmy, Kyagulanyi Tonny, Nuwa Anthony, \\ Magumba Godfrey, Nakwagala Fredrick, Marasciulo Madeleine, \\ Hamade Prudence, and Tibenderana James
}

\section{ABSTRACT}

Background: As COVID-19 disease surges across much of the world, researchers in different settings have a unique opportunity to address the various research priorities that have been identified. The challenges that containment and mitigation strategies present for research, especially in resource limited settings, could be significant and negatively impact the essential contribution of these settings to COVID-19 research.

Objectives: To describe experiences of conducting research during this pandemic, discuss challenges faced and present strategies implemented to address these challenges

Methods: Malaria Consortium recently initiated an observational case series study to assess the magnitude and clinical consequences of coinfection of COVID-19, malaria, and other common infections. This study is being conducted in eight COVID-19 treatment centres in Uganda. Qualitative methods including observations and interviews were utilized to document experiences and mitigating strategies for identified challenges. The main outcomes were a descriptive narrative of experiences conducting this research, discussion of challenges faced, and presentation of strategies implemented to address these challenges.

Results: Expedited ethical review and approval facilitated timely initiation of research activities. The primary clinical care teams at each treatment centre performed all study procedures to minimize infection. Given concerns about fomite transmission, considerations arose on how best to handle consent forms that had been signed or thumb-printed by patients to ensure that both hospital and research staff were not exposed to infection. Consenting severely ill or mentally impaired patients was also a challenge, especially when the next of kin was not available. Patient compensation was done through a mobile money/digital platform to avoid potential risks associated with cash. Patients, health care workers and study staff faced significant psychosocial challenges and anxiety that needed to be addressed.

Conclusions: These experiences demonstrate that more adaptable and innovative approaches may be needed to support the implementation of research activities during this COVID-19 pandemic. This pandemic should also spur institutional review boards and investigators to respond to emerging challenges by updating policies and procedures around research review and approvals, and modifications in research methods.

Keywords: COVID-19 research, ethical review, modifications in research methods.

\section{INTRODUCTION}

As of $16^{\text {th }}$ August 2020, there were more than 21 million confirmed cases of COVID-19 and approximately 761,779 deaths reported globally [1]. In sub-Saharan Africa alone, 47 of the 54 countries had reported COVID-19 cases and at least
Published Online: January 7, 2021

ISSN: $2736-5476$

DOI : $10.24018 /$ clinicmed.2020.2.1.9

Achan Jane*

Malaria Consortium, London, United Kingdom.

(e-mail: j.achan@malariaconsortium.org) Asadu Serwanga

Malaria Consortium, Kampala, Uganda. Aanyu T. Hellen

Mulago National Referral Hospital, Ministry of Health, Kampala, Uganda. Opigo Jimmy

National Malaria Control Division, Ministry of Health, Kampala, Uganda.

Kyagulanyi Tonny

Malaria Consortium, Kampala, Uganda. Nuwa Anthony

Malaria Consortium, Kampala, Uganda.

Magumba Godfrey

Malaria Consortium, Kampala, Uganda.

Nakwagala Fredrick

Mulago National Referral Hospital,

Ministry of Health, Kampala, Uganda.

Marasciulo Madeleine

Malaria Consortium-US, Raleigh, USA.

Hamade Prudence

Malaria Consortium, London, United

Kingdom.

Tibenderana James

Malaria Consortium, London, United Kingdom.

*Corresponding Author
33 of these had ongoing community transmission [1]. Infections that cause pandemics like this novel coronavirus (SARS-CoV-2) have both direct effects related to the morbidity and mortality attributed to the virus and additional significant indirect health effects in affected countries. Such pandemics also present an unprecedented challenge arising 
from the need to rapidly develop new diagnostic, preventive, and therapeutic strategies, as well as the urgent need for clinical and epidemiological studies to enhance understanding of the disease. As SARS-CoV-2 continues to spread across much of the world, the critical importance of research and development in the response to such outbreaks is spotlighted.

As COVID-19 disease surges across much of the world, researchers in different settings have a unique opportunity to address the various research priorities that have been identified [2]. However, pandemic containment and mitigation strategies such as movement restrictions and physical distancing measures have not only caused widespread social and economic disruptions, but also had a major impact on research activities across different settings. The scale of the challenge that this presents for research, especially in resource limited settings, could be significant and negatively impact the essential contribution of these settings to desperately needed COVID-19 research. A recent review of global clinical trials registries, as of March 242020 , identified 536 relevant registered clinical trials of which 332 are COVID-19 related clinical trials [3]. However, very few of these are planned in Africa and other resource limited settings [4]. This observation is also supported by the relatively limited number of COVID-19 publications currently coming from African institutions or researchers. It is therefore vital to understand the unique challenges for conducting research in these settings and identify strategies that could be utilised to address these.

\section{METHODS}

Malaria Consortium recently initiated a prospective observational case series study to assess the magnitude and clinical consequences of co-infection of COVID-19, malaria, and other common infections. This study is being conducted in eight COVID-19 treatment centres in Uganda as a collaboration between Malaria Consortium and the National Malaria Control Division at the Ministry of Health. Qualitative methods including observations and interviews were utilized to document experiences and mitigating strategies for identified challenges. Ethical clearance was obtained from the Mulago Hospital Research and Ethics Committee and Uganda National Council for Science and Technology. In this paper we share our experiences of conducting research during this pandemic, discuss challenges faced and present some of the strategies implemented to address these challenges.

\section{RESULTS AND OBSERVATIONS}

\section{A. Ethical Approval}

Ethical review of studies plays a critical role in protecting the rights, safety, and well-being of research participants and ensuring the scientific integrity of studies [5]. However, the exceptional and complex circumstances of this pandemic called for the use of expedited approaches to ethical review and approval to facilitate timely initiation of research activities. Such approaches need to be supported by existing national or international regulations and guidelines. For this observational study, protocol submission to the Ethics Committee was done at a time when the designated Ethics Committee could not reasonably meet in person to review the study given the lockdown and movement restrictions in the country at the time. However, given the great need to answer scientific questions that can only be done during the context of the pandemic, expedited review was done by the Ethics Committee chair and approval was provided within 48 hours of submission. This approach was guided by existing national guidelines that permit expedited review and approval, with all expedited review decisions presented at the next full Ethics Committee meeting for ratification [6]. Further guidance provided to the research team with this expedited approval was to follow all relevant laws and research guidelines and use only licensed staff. Additionally, all informed consent procedures were to be followed including appropriate compensation of research participants and use of assent where necessary. After four weeks, some of the movement restrictions in Uganda were lifted, this allowed the Ethics Committee to meet with physical distancing. The study protocol and the expedited review decisions were presented at this meeting and final approval was then granted at this full Ethics Committee sitting. With this approach, timely study recruitment was initiated shortly after the first COVID-19 cases were reported in the country which facilitated meaningful accrual going forward. At the national level, approval for research is provided by the Uganda National Council for Science and Technology (UNCST) with submission to UNCST done after EC approval is received. The UNCST was also quite responsive to the pandemic situation and provided timely new guidelines for researchers on the management of research activities. In addition, the UNCST established an online electronic platform for submission of research applications that ensured safe ongoing paperless research review [6].

\section{B. Consent Procedures}

Voluntary informed consent is a prerequisite for a subject's participation in any research study and is an ethical and legal requirement for research involving human participants [7]. The clinical case management of COVID-19 patients in this setting presented some unique challenges to the consent process. The COVID-19 treatment centres are set-up to ensure strict infection prevention and control (IPC) processes are adhered to with a designated red zone which is the highly infectious patient admission area and the green zone the noninfectious largely administrative areas. For this study, written informed consent was obtained for all patients who either signed or thumb-printed on the informed consent documents. This was done by the health facility staff. At the time these consents were being obtained, there was much concern regarding fomite transmission as there was limited data on the duration air droplets on paper could remain infectious, nor what risk this imposed. The critical consideration then was how to handle these consent forms that had been signed or thumb-printed by patients inside the red zone to ensure that both hospital and research staff were not exposed to infection. An option was to leave the consent forms within the red zone, but this also raised concerns about the long-term safety of 
these study documents in the public space in these treatment centres. Our approach to this challenge was to have the hospital staff in protective gear take all completed consent forms out of the red zone either periodically or following each enrollment. The pens and inkpad used for the consenting process were all kept inside the red zone. The consent forms were taken out in biohazard plastic bags that were disinfected outside the red zone and were then subsequently transferred and kept in a second safely sealed biohazard plastic bag and stored in a safe location until such a time when they will be removed and disinfected. Options for disinfection of these consent documents presented yet another challenge for the study team. One consideration was the use of ultraviolet (UV) light automated disinfection systems [8] that had been used by another research group in the same setting for disinfection of such research documents.

Consenting severely ill or mentally impaired patients was also a challenge, especially when the designated next of kin was not available. In these situations, there was an active effort to locate the next of kin, and when this was not possible, the patients were not enrolled. Patients with no designated next of kin were also not enrolled. This limited observation in this category of patients.

\section{Research Staff and Clinical Care Team Interactions}

In the interest of ensuring IPC procedures were followed and strictly adhered to across all recruitment sites, the heads of the COVID-19 treatment centres did not welcome the idea of having multiple research staff from the different research groups entering the various treatment centres across the country. The agreed approach was for the primary clinical care teams at each treatment centre to perform all study procedures including obtaining consent, sample collection and data collection. Ensuring consistency in all study procedures across different sites was therefore critical and this was achieved by training all staff at participating sites on consent procedures, case record form completion and sample collection. This approach was ideal to ensure optimal adherence to IPC procedures and to minimize exposure risk to the multiple individuals from the different research groups. The clinical care staff were fully supportive of this approach and were responsible for scheduling of the different research activities to ensure that compliance was maintained and that there was no work overload. They were also compensated for this extra work.

\section{Patient Compensation}

Existing national guidelines require that research participants be compensated for the time spent participating in studies. This is often clearly indicated in the consent form including the actual compensation amount as a requirement by the Ethics Committee. However, given the potential risk of transmission of infection through direct cash payments to patients in the red zone, such cash payments were generally discouraged while research participants were still hospitalized. This presented yet another challenge as the research team needed to identify an ideal approach to compensating these participants. Compensation was therefore done either through a mobile money (digital) platform for participants that were registered for this service, or who had next of kin registered for this service, and the others only received this compensation at the time of discharge after a negative COVID-19 test was confirmed. Though this approach generally worked well for most participants, a few complaints were received from the group that received the compensation at discharge as this was perceived as delayed compensation. Additional explanations were provided as to why this was important to prevent the risk of exposure resulting from any inadequately handled cash, and these explanations were understood by the patients. Furthermore, given the stigma associated with COVID-19 in these settings, it was important that the patients' personal registration details on these electronic payment platforms where kept confidential. To achieve this, compensation of participants was only managed and done by the research coordinator and not administrative staff.

\section{E. Psychosocial Needs of Patients, Health Care Workers and Study Staff}

Though not a primary objective of the study, the research team was confronted with addressing several issues related to the psychosocial needs of study participants and some health care workers. The telephone contacts provided on the consent form became an unintended helpline to addressing patient concerns about several issues which were mostly outside the scope of the study. One main issue was the duration of hospitalization especially for asymptomatic patients who perceived the long hospital stays as unnecessary and expressed concerns about when they would be discharged. In addition, patients requested for improvement in some aspects of their welfare at these treatment centres including provision of food, timelines for return of COVID-19 test results and improvement in the infrastructure and sanitation at the centres. Others had queries about study compensation and reported significant levels of stress related to confinement within the treatment centres away from family and their economic activities. The study team responded to issues that could be addressed, and also provided feedback to the clinical care teams for follow up and appropriate management. Patients also expressed fear about being stigmatized in the community following discharge. In several cases, when the research team provided transport home for study participants after discharge, they requested to be left at a significant distance from their home to avoid the community members inadvertently discovering that their absence from home may have been due to COVID-19 disease, as the study vehicle was similar to those used by the COVID-19 field surveillance teams.

For the healthcare workers, the main issues raised included concerns about limitations of personal protective equipment (PPE), especially N95 masks; to which the research team responded by supplementing the supplies as needed, and this made a difference in supporting clinical care. Other health worker concerns included stigmatization by members of the communities they served and the challenge of staying away from their families for prolonged periods of time while on duty at the treatment centres. This was a policy at some of the treatment centres, with accommodation and food provided for the health care workers while they were away from their homes. Study staff also reported anxiety related to the fear of 
contracting COVID-19. This was addressed by having optimal IPC training and PPE supplies, frequent testing for SARS-CoV-2 infection, psychosocial support and having flexible working schedules.

\section{F. Issues Pertaining to Feedback of Laboratory Evaluations}

Several other research groups were working at the same COVID-19 treatment centres in the country as each treatment centre had a limited number of infected and hospitalized patients. As a result of overlapping research activities, patients underwent multiple assessments and also had samples collected for different evaluations including routine clinical laboratory evaluations, study specific sample collections and COVID-19 follow-up diagnostic testing. Several patients raised concerns about delayed return of the COVID-19 test results which they thought contributed to their delayed discharge. In addition, given that they had been assessed by multiple groups, the patients also expected formal feedback of all the laboratory results for the different samples collected. This request for laboratory results was particularly apparent in centres with multiple research groups operating with different samples collected and varying timelines for laboratory evaluations and return of results. However, the challenge herein is that some of the studies may not have been able to return results before patient discharge given that some of the tests were not done in real-time. Most test results needed for clinical care were available immediately which was important, but the other research specific specialised assays may have had longer turnaround times that needed to be communicated better with the patients. The specific research groups through the health facility clinical care teams could have provided better explanations for the different assays and turn-around times to the patients. Better coordination between the research groups could have streamlined such operational aspects as well. In one of the larger treatment centres, the three research groups had a collaborative agreement in place that streamlined communication and research procedures.

\section{G. Maintaining Team Communication Remotely}

Team communication is an essential aspect of conducting research and its vital that it be maintained to ensure efficient research implementation. Effective communication ensures that investigators provide quality training and appropriate supervision for staff involved in research implementation. For this study, training of staff and study initiation was done on-site at each of the participating treatment centres. This was done following standard IPC procedures and national guidelines for prevention of transmission of the SARS-CoV2. However, subsequent communication with the staff and research teams was done through virtual meetings using Zoom or two-way telephone calls. These virtual meetings were used to share study progress and discuss any issues arising. Whereas these communication modalities differed from standard approaches used in these settings, it was fully embraced by the staff as a vital strategy to ensuring timely decision-making and responsible conduct of research. However, limited internet coverage at some of the sites and heavy clinical care schedules presented significant challenges in some areas.

\section{DISCUSSION}

Global health emergencies trigger profound immediate and long-lasting consequences at both national and international levels, as evidenced by the Zika virus outbreak, the westAfrican Ebola outbreak [9] and the ongoing COVID-19 pandemic. In these situations, the ability to conduct research is particularly important, especially where the pathogens are novel, the corresponding therapeutics are non-existent or in need of validation, and epidemiological profiles are unknown. During such emergencies, whereas the need to learn as much as possible as quickly as possible [10] is of utmost importance, without overburdening an already stretched clinical service, the relationship between response and research gives rise to several ethical and regulatory challenges. We share experiences of conducting a clinical study in the context of the COVID-19 pandemic and strategies that were useful in addressing the identified challenges.

\section{A. Ethical Review}

Given that ethical review is required before the launch of any biomedical research study involving human subjects [11], the current COVID-19 pandemic presents exceptional circumstances for which special considerations for modifying the ethics review process are warranted. Considering the magnitude of the pandemic, its contagiousness, the burden imposed on health systems, and the limited time within which investigators can answer relevant research questions, timely ethical review is vital. With the urgent need for new scientific knowledge in such situations, any gaps in existing guidelines and regulations for scientific research management in the context of major public health emergencies need to be addressed. As the number of research studies involving human subjects on COVID-19 rises in different settings, ethical review committees need to be more responsive in terms of reviewing speed and addressing special ethical considerations during the pandemic. A major concern, especially in Africa, is that the existing ethical review systems and regulations may be limited in their ability to meet the demand for a prompt and efficient review and approval of studies [10]. For our study, the timely review and expedited approval was vital in ensuring timely initiation of study activities. This approval process was supported by existing national regulations in Uganda. Addressing limitations in local research ethics review and scientific capacity and providing ethics review in time-sensitive circumstances should be a key priority in other settings as well. Supportive regulations need to be promoted to support research during such complex emergencies. Options for virtual meetings to review and approve protocols should also be explored and embraced.

\section{B. Participant Compensation}

Compensation of research participants is generally an acceptable practice [12] in several settings including Uganda. Whereas compensation in our study was done for the time of 
participation in the study, compensation in other studies may be handed out as refunds for expenses incurred by participants, level of effort, and any inconvenience associated with research participation [12]. In the context of this highly contagious pandemic, the modalities of direct cash transactions, which are the norm in many settings, become a challenge especially for effective infection prevention and control. This called for innovative approaches to providing compensation such as electronic and digital payment platforms which were used in this study. This approach feeds into global calls to promote online/digital payment platforms especially in the context of minimising exposure risk from direct cash transactions during this COVID-19 pandemic. The implementation of such approaches may however be limited in remote and rural areas that may not have access to such platforms. However, with the wide network of mobile telecommunication coverage, mobile money platforms could become an attractive option for compensation in similar studies. For participants with no access to this modality of payment, delays in compensation resulted in dissatisfaction which was addressed by patient education.

\section{Involvement of Host Health Facility Staff}

Involvement of treatment centre staff in the study implementation improved collaboration between the health care workers and research team, and also enhanced ownership and acceptance of the study within the treatment centres. Study observations also fed back into clinical care as some clinical laboratory results not routinely done by the health facilities contributed to patient management. Overall, this approach to data collection was beneficial in minimising exposure risk and transmission of infection across sites by limiting access to the red zone by various research groups. This also promoted better coordination of different groups working in the same settings. In some treatment centres, collaborative research agreements were drawn to enhance efficiency of operations, avoid duplication and promote data sharing where applicable. Such coordination of research efforts and data sharing could also be a role played by the ECs or similar research committees.

\section{Informed Consent Processes}

The process of obtaining informed consent and the handling of the consent documents was also challenging. Whereas the approach used in this setting seemed reasonable, it is difficult to determine what the best practice is in such situations. Ethical consideration for obtaining consent in such settings with critically ill and highly infectious patients need to be reviewed.

To understand optimal approaches for such situations requires collaboration and sharing of experiences amongst key stakeholders, different research groups involved in similar work and patient groups as well [9]. Such collaborations have been useful for identification and formulation of best practice guidelines during previous outbreaks like the Zika virus epidemic [13] and the Ebola epidemic [14]. However, challenges of reaching consensus in the development of best practice exist as different groups will inevitably reflect their own versions of best practice largely informed by their experiences. In general, such public health emergencies require flexible applications of ethical principles [15] and different approaches to consent have been used previously with some studies using short written documents [16] or verbal consent after patients were presented with the study's purpose and procedures [17], [18]. Any such flexibility or adaptations in the application of ethical principles should uphold, and not compromise, the standards for the rights and protection of research subjects. Optimal approaches to the dissemination of any best practices are also necessary to ensure that these are readily available on a publicly accessible platform.

\section{E. Psychosocial and mental health issues}

In addition to ensuring compliance with existing research regulations and guidelines, and maintaining effective IPC strategies, its vital that the psychosocial and mental health needs of patients, health care workers and research staff are addressed. Whereas mental health and psychosocial support constitutes a key pillar of management of patients with COVID-19 in this setting, it is not clear how accessible these services are to the health care providers in the same settings. Its therefore important that staff are aware of where and how they can access mental health and psychosocial support services and health facility managers and team leaders should facilitate access to such services [19]. Keeping all staff protected from chronic stress and poor mental health is critical during such times. Flexible working schedules and rotation of staff from higher-stress to lower-stress roles is also helpful in addressing mental health issues [19]. Finally, good quality communication and accurate information should be provided to all staff to relieve any anxiety and fear.

\section{CONCLUSION}

In conclusion, whereas several challenges arise from the conduct of research during global health emergencies, the COVID-19 pandemic is compelling the scientific community to innovate solutions to standard research practices which are difficult to meet. The pandemic should spur institutional review boards and investigators to respond to emerging challenges by updating policies and procedures around research review and approvals, consent, assessments, compensation, and modifications in research methods. The experiences shared herein demonstrate that more adaptable and innovative approaches may be needed to support the implementation of research activities during this COVID-19 pandemic. Overall, these approaches could be generalizable to other similar settings to support timely implementation of clinical research in such complex emergencies. We are hopeful that the necessary changes in policies and procedures highlighted during this pandemic will have a positive and lasting impact on clinical research in similar situations.

\section{ACKNOWLEDGMENT}

We would like to thank the health care workers at all the COVID-19 treatment centres for their contribution to the study. Special gratitude to the patients for their contributions to the study. We acknowledge the immense support from 
Charles Nelson, the Chief Executive of Malaria Consortium and other staff including Kevin Chaudhary, Israel Busiko, Sadi Mwanga and Michael Okumu for their support.

\section{CONFLICTS OF INTEREST}

Drs Helen T. Aanyu and Fredrik Nakwagala are members of the Mulago Hospital Research and Ethics but did not participate in decisions pertaining this study. The other authors declare no conflicts of interest.

\section{REFERENCES}

[1] WHO. Coronavirus disease (COVID-19) Situation Report - 209. 2020

[2] WHO. A Coordinated Global Research Roadmap: 2019 Novel Coronavirus. 2020.

[3] Maguire BJ, Guerin PJ. A living systematic review protocol for COVID-19 clinical trial registrations. Wellcome Open Res. 2020;5:60.

[4] Coalition C-CR. Global coalition to accelerate COVID-19 clinica research in resource-limited settings. The Lancet. 2020;395(10233):1322-5.

[5] Ma X, Wang Y, Gao T, He Q, He Y, Yue R, et al. Challenges and strategies to research ethics in conducting COVID-19 research. J Evid Based Med. 2020;13(2):173-7.

[6] UNCST UNCfSaT-. National Guidelines for Research involving Humans as Research Participants. Kampala, Uganda2014.

[7] CIOMS CfIOfMS-. International Ethical Guidelines for Health-Related Research Involving Humans. Geneva, Switzerland; 2016 1st September 2020.

[8] Weber DJ, Kanamori H, Rutala WA. 'No touch' technologies for environmental decontamination: focus on ultraviolet devices and hydrogen peroxide systems. Curr Opin Infect Dis. 2016;29(4):424-31.

[9] Sethi N. Research and Global Health Emergencies: On the Essential Role of Best Practice Public Health Ethics. 2018;11(3):237-50.

[10] WHO. Guidance for Managing Ethical Issues in Infectious Disease Outbreaks. Geneva, Switzerland; 2016.

[11] Ma XW, Y. Gao, T., He Q, He Y, Yue R, You F, Tang J. Challenges and strategies to research ethics in conducting COVID-19 research. J Evid Based Med. 2020;13(2):173-7.

[12] Grady C. Payment of clinical research subjects. J Clin Invest. 2005;115(7):1681-7.

[13] Pregnancy EWGoZRa. Pregnant Women \& the Zika Virus Vaccine Research Agenda: Ethics Guidance on Priorities, Inclusion, and Evidence Generation. London: Wellcome Trust.; 2017 3rd September 2020.

[14] WHO. Meeting of the Ethics Working Group on Ebola Interventions. Geneva, Switzerland; 201420 - 21 October.

[15] Saxena A, Gomes M. Ethical challenges to responding to the Ebola epidemic: the World Health Organization experience. Clin Trials. 2016;13(1):96-100

[16] Konde MK, Baker DP, Traore FA, Sow MS, Camara A, Barry AA, et al. Interferon beta-1a for the treatment of Ebola virus disease: A historically controlled, single-arm proof-of-concept trial. PLoS One. 2017;12(2):e0169255.

[17] Schieffelin J, Moses LM, Shaffer J, Goba A, Grant DS. Clinical validation trial of a diagnostic for Ebola Zaire antigen detection: Design rationale and challenges to implementation. Clin Trials. 2016;13(1):6672.

[18] Sissoko D, Laouenan C, Folkesson E, M'Lebing AB, Beavogui AH, Baize S, et al. Experimental Treatment with Favipiravir for Ebola Virus Disease (the JIKI Trial): A Historically Controlled, Single-Arm Proofof-Concept Trial in Guinea. PLoS Med. 2016;13(3):e1001967.

[19] WHO. Mental health and psychosocial considerations during the COVID-19 outbreak 2020 [updated 18th March. Available from: https://www.who.int/docs/default-source/coronaviruse/mental-healthconsiderations.pdf. 